\title{
Validez discriminante de la Escala de Conductas de Autocuidado para Psicólogos Clínicos
}

\section{Discriminant validity of Self-Care Behaviours Scale for Clinical Psychologists}

\author{
Cristóbal Guerra Vio ${ }^{1}$ \\ Universidad Santo Tomás, Chile \\ (Rec: Agosto 2011 - Acep: Octubre 2011)
}

\begin{abstract}
Resumen
El objetivo del presente estudio es evaluar la validez discriminante de la Escala de Conductas de Autocuidado para Psicólogos Clínicos (EAP; Guerra, Morales, Rodríguez y Betta, 2008). Esta escala mide la frecuencia con que los psicólogos clínicos ejecutan conductas de autocuidado. En estudios previos la EAP ha mostrado adecuados índices de fiabilidad y validez (factorial y convergente), empero no existen antecedentes respecto de su poder de discriminación. Para dar respuesta a este vacío de conocimiento se aplica la EAP a dos grupos de psicólogos, sin estrés traumático secundario $(n=126)$ y con estrés traumático secundario $(n=70)$, y se comparan sus puntajes. Los resultados respaldan la validez discriminante de la EAP, ya que los psicólogos sin estrés traumático secundario obtienen puntajes significativamente mayores en la escala que aquellos psicólogos que presentan dicho trastorno. Estos resultados se suman a los antecedentes que otorgan validez a la escala.

Palabras clave: autocuidado, psicólogos clínicos validez.
\end{abstract}

\begin{abstract}
The purpose of the present study is to evaluate the discriminant validity of the Self-Care Behaviors Scale for Clinical Psychologists (EAP; Guerra, Morales, Rodríguez and Betta, 2008). This scale measures the frequency with which the clinical psychologists execute self care behaviors. In previous studies the EAP it has showed suitable indexes of reliability and validity (factorial and convergent), nevertheless precedents do not exist respect of his power of discrimination. To give response to this emptiness of knowledge the EAP is applied to two groups of psychologists; without secondary traumatic stress $(n=126)$ and with secondary traumatic stress $(n=70)$, and his scores are compared. The results endorse the discriminant validity of the EAP since the psychologists without traumatic secondary stress obtain scores significantly major in the scale that those psychologists who present the above mentioned disorder. These results add to the previous precedents that grant validity to the scale. Key words: self -care, clinical psychologists and validity.
\end{abstract}

\footnotetext{
Correspondencia a: Cristóbal Guerra Vio. Dirección: Escuela de Psicología, Universidad Santo Tomás, Viña del Mar, Chile. Tel: 2448022. E-Mail: cguerravio@yahoo.es.
} 


\section{Introducción}

El constructo autocuidado ha sido utilizado por diversos autores (Figley, 2002; Hesse, 2002; Pearlman \& Saakvitne, 1995; Richardson, 2001; Saakvitne \& Pearlman, 1996) para dar cuenta de las acciones que realiza un profesional de ayuda para prevenir y tratar los síntomas del desgaste laboral.

Hidalgo y Moreira (2000) indican que el autocuidado corresponde a un constructo estrechamente ligado a lo práctico, ya que refleja las diferentes y variadas estrategias concretas que son implementadas al interior de los equipos profesionales para hacer frente al desgaste laboral. Debido a lo anterior ha existido dificultad para operacionalizar el constructo autocuidado en profesionales, lo que se ve reflejado en la ausencia de instrumentos para medir este consructo en este tipo de población (Guerra, Morales, Rodríguez \& Betta, 2008).

La ausencia de operacionalización del autocuidado se constituye como un problema de relevancia pues al no existir parámetros objetivos para medir el constructo, se hace difícil evaluar la efectividad de programas de autocuidado en población de profesionales. Esta situación es preocupante, de hecho, distintos autores han documentado las consecuencias del desgaste laboral. Por ejemplo, Morales, Pérez y Menares (2003) afirman que los profesionales desgastados presentan un aumento en el uso de licencias médicas y en el ausentismo laboral. Por su parte, Beaton y Murphy (1995) sostiene que los profesionales desgastados presentan un mayor nivel de consumo de drogas y desarrollan trastornos psicológicos con mayor frecuencia que los profesionales que no lo están. Finalmente, se ha señalado que los profesionales desgastados tienden a disminuir su rendimiento laboral (Gentry, 2003; Salston \& Figley, 2003; Valent, 2002).

Por otro lado, se ha establecido que los psicólogos clínicos y terapeutas son una población altamente vulnerable de presentar desgaste laboral (Arón \& Llanos, 2001; Barudy, 2000; Figley, 1999; McCann \& Pearlman, 1990; Moreno-Jiménez, Morante, Garrosa \& Rodríguez, 2004; Sabo, 2006).

Motivados por esta situación se elaboró la Escala de conductas de autocuidado para psicólogos clínicos (EAP; Guerra et al., 2008). Esta escala incluye una serie de conductas y condiciones de autocuidado que en investigaciones anteriores han presentado relaciones inversas con diferentes medidas de desgaste laboral en psicólogos clínicos (Betta, Morales, Rodríguez \& Guerra, 2007; Guerra, 2008).
Estudios previos han apoyado la validez de la escala. Específicamente se ha establecido validez de contenido de los reactivos de la escala mediante el juicio de expertos (Betta et al., 2007) y se ha apoyado la validez de constructo de la EAP al obtenerse una estructura unifactorial que agrupa todos los reactivos de la escala, al darse relaciones significativas inversas con constructos teóricamente relacionados con el autocuidado, como la depresión, ansiedad, estrés traumático secundario y síndrome de burnout, y al obtener relaciones directas y significativas con una puntuación del nivel de calidad de vida (Guerra, et al, 2008; Guerra, Mujica, Nahmias \& Rojas, 2011). Además, la fiabilidad de la EAP ha sido adecuada en los estudios recién citados (valores alfa de Cronbach superiores a 0,74).

No obstante lo anterior, no existen estudios que evalúen la capacidad de discriminación de la EAP. Por lo tanto, este estudio busca determinar si la EAP posee adecuados índices de validez discriminante. Para ello se realiza un análisis de la capacidad de la escala para discriminar entre profesionales que presenten distintos niveles de desgaste laboral.

\section{Método}

\section{Participantes}

Se trabajó con una muestra intencionada compuesta por 196 psicólogos clínicos chilenos que al momento de la investigación se desempeñaban profesionalmente en la Región de Valparaíso. Todos los sujetos de la muestra dieron su consentimiento para participar de la investigación.

Los participantes, con edades entre 23 y 77 años $(\mathrm{M}=34,71 ; \mathrm{DT}=9,34)$, fueron mayoritariamente de género femenino (74,6\%). En promedio llevaban 7,27 años $(\mathrm{DT}=7,13)$ trabajando como psicólogos y 6,48 años $(\mathrm{DT}=6,85)$ trabajando como psicólogos clínicos.

Los encuestados, en promedio, presentaron niveles bajos de desgaste laboral (estrés traumático secundario; $\mathrm{M}=34,84 ; \mathrm{DT}=11,3)$. No obstante, es importante destacar que estos psicólogos fueron divididos en dos grupos según los niveles de desgaste laboral (estrés traumático secundario) que presentaron.

Un primer grupo estuvo conformado por 126 psicólogos con niveles de estrés traumático secundario por debajo del puntaje de corte de la escala utilizada para medir el trastorno. Un segundo grupo estuvo conformado por los 70 psicólogos restantes que presentaron puntajes de estrés traumático secundario por sobre 
el punto de corte de la Escala de estrés traumático secundario.

Ambos grupos exhibieron composiciones similares en edad, género, años trabajando como psicólogos y años trabajando como psicólogos clínicos. La única diferencia estadísticamente significativa entre los grupos fue su nivel de estrés traumático secundario. La tabla 1 muestra las características de la muestra total y luego los resultados del contraste estadístico realizado entre ambos grupos en cada una de las variables aquí señaladas.

\section{Instrumentos}

Se utilizó una batería de tres instrumentos:

Escala de Conductas de Autocuidado para Psicólogos Clínicos (EAP; Guerra et al., 2008). Corresponde a una escala autoaplicada que permite medir la frecuencia con que los psicólogos clínicos ejecutan conductas de autocuidado (ítems 1, 2, 4, 5, 6, 7,8 y 9) y la frecuencia con que su ambiente laboral favorece condiciones de autocuidado (ítems 3 y 10). Las opciones de respuesta varían entre 0 (nunca) y 4 (muy frecuentemente). La sumatoria de los 10 ítems de la escala permite la obtención de un puntaje total de autocuidado. Puntajes entre 0 y 7 puntos indican que el psicólogo nunca practica el autocuidado, entre 8 y 15 indican que lo practica casi nunca, entre 16 y 23 puntos se considera que lo practica ocasionalmente, entre 24 y 31 puntos que la persona frecuentemente utiliza las estrategias, y puntajes totales sobre 32 puntos indican que el psicólogo utiliza el autocuidado тиy frecuentemente. Para esta investigación la EAP presentó adecuados índices de fiabilidad (alfa de Cronbach de 0,78).

Escala de Estrés Traumático Secundario (EETS; Bride, Robinson, Yegidis \& Figley, 2004; adaptada a Chile por Guerra \& Saiz, 2007). Es una escala autoaplicada de 17 ítems que mide síntomas intrusivos, evitativos y de activación fisiológica en profesionales de ayuda. La escala de respuestas varía entre 1 (nunca) y 5 (con mucha frecuencia). De la sumatoria de los ítems se obtiene el puntaje total de estrés traumático secundario (en adelante ETS). Bride (2007) indica que puntajes entre 17 y 27 puntos representan una sintomatología muy baja, entre 28 y 37 puntos se considera que la sintomatología es baja, entre los 38 y 43 puntos la sintomatología es moderada, entre 44 y 48 puntos los niveles de ETS son altos y sobre los 48 severos. Además, el autor señala que el puntaje de corte se establece en los 38 puntos. En esta investigación, la EETS mantuvo una adecuada consistencia interna (alfa de Cronbach de 0,93).

Cuestionario de variables sociodemográficas. Se elaboró un breve cuestionario para obtener datos descriptivos de los participantes. Específicamente se incluyeron reactivos destinados a conocer la edad, el género, los años de experiencia como psicólogo y los años de experiencia como psicólogo clínico.

Tabla 1. Características de la muestra total y comparación de las características de los psicólogos con y sin estrés traumático secundario.

\begin{tabular}{|c|c|c|c|c|}
\hline Variable & $\begin{array}{l}\text { Muestra total } \\
(\mathrm{n}=196)\end{array}$ & $\begin{array}{l}\text { Psicólogos sin desgaste } \\
(\mathrm{n}=126)\end{array}$ & $\begin{array}{l}\text { Psicólogos desgastados } \\
(\mathrm{n}=70)\end{array}$ & $\begin{array}{l}\text { Diferencia (sin desgaste } \\
\text { vs. desgastados) }\end{array}$ \\
\hline Edad (años) & $\begin{array}{l}\mathrm{M}=34,71 \\
\mathrm{DT}=9,34\end{array}$ & $\begin{array}{l}\mathrm{M}=34,56 \\
\mathrm{DT}=9,65\end{array}$ & $\begin{array}{l}\mathrm{M}=35,00 \\
\mathrm{DT}=8,834\end{array}$ & $\begin{array}{l}t=-0,31 \\
p=0,75\end{array}$ \\
\hline \multicolumn{5}{|l|}{ Género } \\
\hline Femenino & $74,6 \%$ & $76,9 \%$ & $62,8 \%$ & $\chi 2=2,98$ \\
\hline Masculino & $25,4 \%$ & $23,1 \%$ & $37,2 \%$ & $\mathrm{p}=0,08$ \\
\hline Años como psicólogo & $\mathrm{M}=7,27 \mathrm{DT}=7,13$ & $\mathrm{M}=6,71 \mathrm{DT}=7,18$ & $\mathrm{M}=8,27 \mathrm{DT}=6,99$ & $\begin{array}{l}t=-1,47 \\
p=0,14\end{array}$ \\
\hline $\begin{array}{l}\text { Años como psicólogo } \\
\text { clínico }\end{array}$ & $\begin{array}{l}\mathrm{M}=6,48 \\
\mathrm{DT}=6,85\end{array}$ & $\mathrm{M}=6,03 \mathrm{DT}=6,80$ & $\mathrm{M}=7,30 \mathrm{DT}=6,92$ & $\begin{array}{l}t=-1,24 \\
p=0,21\end{array}$ \\
\hline Niveles de ETS & $\begin{array}{l}\mathrm{M}=34,84 \\
\mathrm{DT}=11,3\end{array}$ & $\mathrm{M}=28,06 \mathrm{DT}=5,72$ & $\begin{array}{l}M=47,06 \\
\mathrm{DT}=8,36\end{array}$ & $\begin{array}{l}t=-18,78 \\
p=0,00\end{array}$ \\
\hline
\end{tabular}

Nota: $\mathrm{t}=$ prueba $\mathrm{t}$ para muestras independientes y $\chi 2=$ chi cuadrado. 
Gráfico 1. Frecuencia de conductas de autocuidado en los dos grupos de psicólogos.

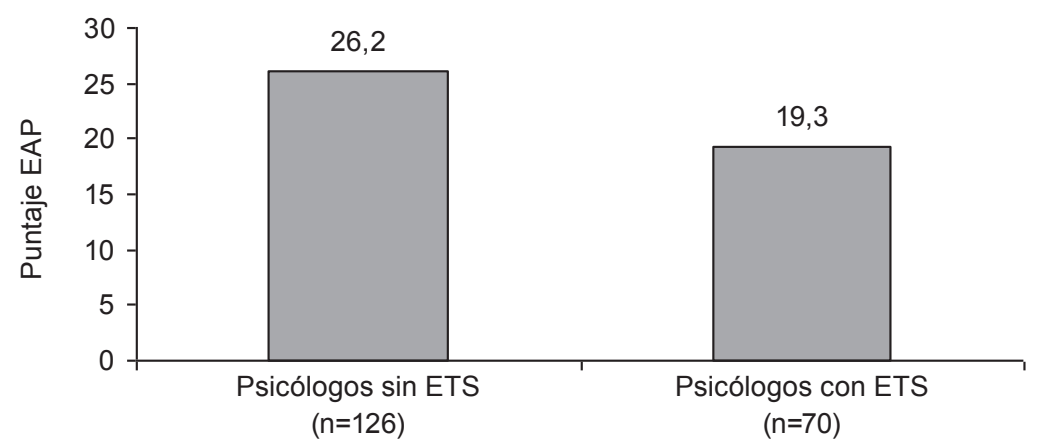

$t=8,733 ; p=0,00$

\section{Procedimiento}

Mediante entrevista personal y/o telefónica se les solicitó a psicólogos clínicos de la Región de Valparaíso de Chile que participaran de la investigación. A los psicólogos que accedieron a participar se les entregó personalmente la batería de instrumentos y se les dio un plazo de dos semanas para responder. Los instrumentos respondidos completamente fueron procesados en el programa estadístico SPSS.

\section{Resultados}

Para evaluar la validez discriminante de la escala se procedió a clasificar a los participantes en dos grupos según el nivel de estrés traumático secundario que presentaban. El grupo 1 quedó conformado por los 126 psicólogos que no presentaban el trastorno y el grupo 2 quedó conformado por los 70 psicólogos que sí lo presentaban. Posteriormente se evaluó, mediante la prueba t de comparación de medias, la hipótesis de que la media de las puntuaciones en la EAP del primer grupo eran superiores a las del segundo grupo.

Tal como era de esperarse, los psicólogos clínicos que no poseen ETS obtuvieron puntajes significativamente mayores en la EAP que aquellos psicólogos que sí están desgastados ( $\mathrm{t}=8,733 ; \mathrm{p}=0,00)$. Específicamente los psicólogos sin ETS puntúan 26,20 $(\mathrm{DT}=5,09)$ en la EAP, lo que indica que frecuentemente

Gráfico 2. Frecuencia de conductas de autocuidado en psicólogos, según el nivel de ETS.

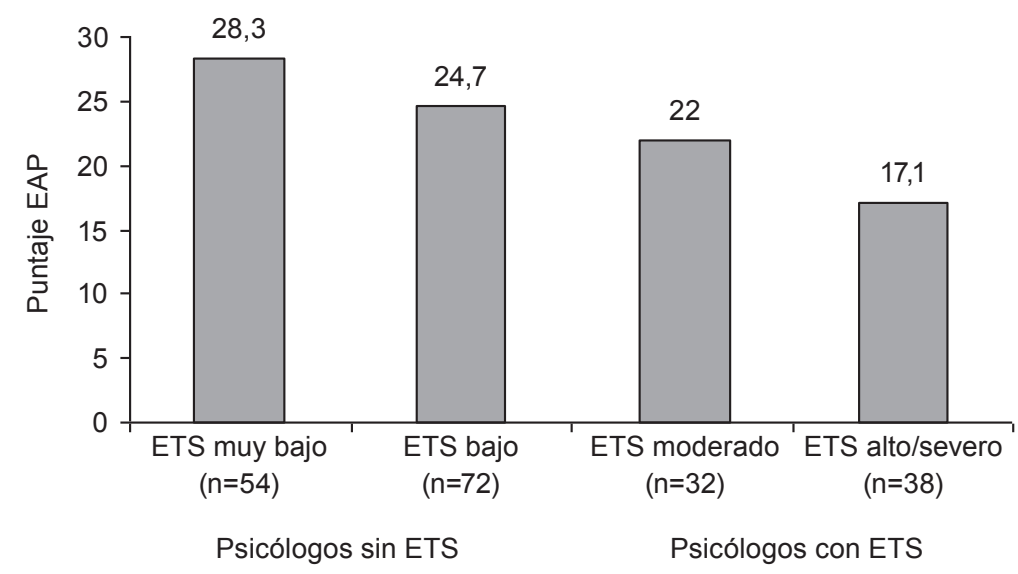

$F=41,137 ; p=0,00$ 
ejecutan las conductas de autocuidado medidas, en cambio los psicólogos con ETS puntúan 19,34 (DT= $5,55)$ en la EAP, lo que indica una práctica ocasional del autocuidado (ver gráfico 1). Estos resultados apoyan la validez discriminante de la EAP.

Para un análisis más detallado de la capacidad de discriminación de la escala se realizó una nueva clasificación de los participantes. Para conformar los grupos se tomó como base la propuesta de clasificación de los puntajes de ETS medidos con la EETS (Bride, 2007).

Se formaron cuatro grupos. El primero estuvo integrado por los psicólogos que presentaron "muy bajos" niveles de ETS ( $\mathrm{n}=54)$; el segundo incluyó a los de "bajos" niveles de ETS $(n=72)$; el tercero consideró a aquellos con "moderados" índices de ETS $(n=32)$; finalmente, el cuarto grupo estuvo compuesto por los psicólogos con sintomatología de ETS “alta" y "severa" $(\mathrm{n}=38)$.

Para comparar los puntajes en la EAP de estos 4 grupos se utilizó el análisis de varianza (Anova de un factor). Los resultados de este análisis apoyan la validez de la escala, ya que sugieren que la EAP discrimina adecuadamente entre los psicólogos con distintos niveles de ETS. Específicamente, los psicólogos con "muy bajos" niveles de ETS puntuaron en promedio 28,26 (DT= 5,58) puntos en la EAP, los con "bajos" niveles de ETS 24,65 (DT=4,10), los de niveles "moderados" 22,00 (DT=4,72) y los de niveles "altos o severos" de ETS obtuvieron en promedio
17,11 (DT=5,25) puntos en la EAP. Estas diferencias entre los cuatro grupos son estadísticamente significativas $(\mathrm{F}=41,137 ; \mathrm{p}=0,00)$ (ver gráfico 2 ).

Luego, para ver entre qué grupos específicos existían diferencias estadísticamente significativas se aplicó la prueba de Scheffé. Los resultados de este análisis aroojan que, en su mayoría, los 4 grupos presentan diferencias estadísticamente significativas entre sí. La única excepción la constituye la ausencia de diferencia significativa entre los grupos con "baja" y "moderada" sintomatología de ETS. El detalle puede observarse en la tabla 2.

\section{Discusión}

El objetivo de este estudio fue evaluar la validez discriminante de la EAP. Para ello se comparó el puntaje en la escala obtenido por psicólogos clínicos que presentaban distintos niveles de un tipo específico de desgaste laboral (el ETS).

Los resultados confirmaron la hipótesis de que la EAP presentaría adecuados índices de validez discriminante, ya que en los distintos análisis estadísticos realizados quedó claramente establecido que la EAP discrimina adecuadamente. Específicamente, los psicólogos que no estaban desgastados presentaron puntuaciones promedio en la EAP significativamente mayores que aquellos que sí estaban desgastados. Esto confirma que la EAP efectivamente mide el constructo autocuidado.

Tabla 2. Comparaciones múltiples de las medias obtenidas en la EAP por los 4 grupos en estudio, mediante la prueba de Scheffé.

\begin{tabular}{llccc}
\hline (I) Nivel de ETS & (J) Nivel de ETS & $\begin{array}{c}\text { Diferencia de } \\
\text { medias (I-J) }\end{array}$ & Error típico & Sig. \\
\hline \multirow{2}{*}{ Sintomatología muy baja } & Sintomatología baja & $3,606\left(^{*}\right)$ & 0,878 & 0,001 \\
& Sintomatología moderada & $6,259\left(^{*}\right)$ & 1,087 & 0,000 \\
& Sintomatología alta o severa & $11,154\left(^{*}\right)$ & 1,032 & 0,000 \\
& Sintomatología muy baja & $-3,606\left(^{*}\right)$ & 0,878 & 0,001 \\
Sintomatología baja & Sintomatología moderada & 2,653 & 1,036 & 0,091 \\
& Sintomatología alta o severa & $7,548\left(^{*}\right)$ & 0,977 & 0,000 \\
& Sintomatología muy baja & $-6,259\left(^{*}\right)$ & 1,087 & 0,000 \\
Sintomatología moderada & Sintomatología baja & $-2,653$ & 1,036 & 0,091 \\
& Sintomatología alta o severa & $4,895\left(^{*}\right)$ & 1,170 & 0,001 \\
& Sintomatología muy baja & $-11,154\left(^{*}\right)$ & 1,032 & 0,000 \\
Sintomatología alta o severa & Sintomatología baja & $-7,548(*)$ & 0,977 & 0,000 \\
& Sintomatología moderada & $-4,895\left(^{*}\right)$ & 1,170 & 0,001 \\
\hline
\end{tabular}

* La diferencia entre las medias es significativa al nivel 0,01 . 
Estos hallazgos se suman a los antecedentes que otorgan validez a la escala (Betta et. al, 2007; Guerra et. al, 2008; Guerra et. al, 2011). Por lo tanto, se concluye que la EAP permite evaluar la frecuencia de conductas de autocuidado en psicólogos clínicos, por lo que puede ser un aporte importante en la evaluación de programas destinados a aumentar la práctica del autocuidado en este tipo de población.

También es importante señalar que los resultados de la presente investigación permiten reflexionar en torno a la trascendencia del autocuidado en los psicólogos clínicos, pues aquellos psicólogos que menos cuidaban de sí mismo eran los más desgastados y viceversa. En este sentido se considera que el autocuidado debiera constituirse como un tema de especial relevancia dentro de la práctica cotidiana de la psicología clínica, ya que las consecuencias del desgaste laboral pueden afectar tanto al propio profesional (de forma directa) como a sus clientes (indirectamente al recibir una atención deficiente). Por tanto, el autocuidado es potencialmente beneficioso para ambas partes, de modo que debiera ser una obligación ética, del propio psicólogo y de los directivos de la organización donde este trabaje, asociada a la preocupación por el cuidado del terapeuta y de sus clientes.

\section{Referencias}

Arón, A. y Llanos, M. (2001). Desgaste profesional. En A. Arón, (Ed.), Violencia en la familia. Programa de intervención en red: La experiencia de San Bernardo (pp. 67-103). Santiago: Galdoc. Barudy, J. (2000). Maltrato infantil, ecología social: prevención y reparación. Barcelona: Paidós.

Beaton, R. D., y Murphy, S. A. (1995). Working with people in crisis: Research implications. En Ch. Figley (Ed.). Compassion fatigue: Coping with secondary traumatic stress disorder in those who treat the traumatized (pp. 51-81). New York: Brunner/Mazel Publishers.

Betta, R., Morales, G., Rodríguez, K. y Guerra, C. (2007). La frecuencia de emisión de conductas de autocuidado y su relación con los niveles de estrés traumático secundario y depresión en psicólogos clínicos. Pensamiento Psicológico, 9, 9-19.

Bride, B. E. (2007). Prevalence of secondary traumatic stress among social workers. Social Work, 52(1), 63-70.

Bride, B. E., Robinson, M. M., Yegidis, B. y Figley, C. R.. (2004). Development and validation of the Secondary Traumatic Stress Scale. Research on Social Work Practice, 14, 27-35.

Figley, Ch. R. (1999). Compassion fatigue: Toward a new understanding of the cost of caring. En B. H. Stamm (Ed.), Secondary traumatic stress: Self-care issues for clinicians, researches, and educators (pp. 3-28). Lutherville, MD: Sidran Press.

Figley, Ch. R. (2002b). Compassion fatigue: Psychoterapist's chronic lack of self care. Journal of Clinical Psychology, 58(11), 1433-1441.

Gentry, J. E. (2003). Desgaste por empatía: el desafío de la transformación. Revista de Psicotrauma para Iberoamérica, 2(2), 4-17.
Guerra, C. y Saiz, J. L. (2007). Examen psicométrico de la Escala de Estrés Traumático Secundario: un estudio en profesionales chilenos. Psicología Conductual, 15, 441-456.

Guerra, C. (2008). Descripción de los niveles de estrés traumático secundario y su relación con las prácticas de autocuidado en psicólogos clínicos chilenos que se desempeñan en diferentes contextos laborales: análisis desde la Terapia Centrada en las Soluciones. Tesis de Postítulo no publicada. Centro de Especialistas en Intervención Estratégica (MIP), Santiago, Chile.

Guerra, C., Rodríguez, K., Morales, G., y Betta, R. (2008). Validación preliminar de la escala de conductas de autocuidado para psicólogos clínicos. Psykhe 17 (2), 67-77.

Guerra, C., Mujica, A., Nahmias, A. y Rojas, N. (2011). Análisis psicométrico de la Escala de conductas de autocuidado para psicólogos clínicos. Revista Latinoamericana de Psicología 43(2), 115-124.

Hesse, A. (2002). Secondary trauma: How working with trauma survivors affects therapists. Clinical Social Work Journal, 30, 293-309.

Hidalgo, N. y Moreira, E. (2000). Autocuidado, Trastorno psicológico y rotación de equipos de salud mental. Santiago, Chile: Universidad de Chile, Departamento de Psicología.

McCann, I. L., y Pearlman, L. A. (1990). Vicarious traumatization: A framework for understanding the psychological effects of working whit victims. Journal of Traumatic Stress, 3(1), 131-149.

Morales, G., Pérez, J. y Menares, M. (2003). Procesos emocionales de cuidado y riesgo en profesionales que trabajan con el sufrimiento humano. Revista de Psicología Universidad de Chile, 12(1), 9-25.

Moreno-Jiménez, B., Morante, M. E., Garrosa, E., y Rodríguez, R. (2004). Estrés traumático secundario: el coste de cuidar el trauma. Psicología Conductual, 12(2), 215- 231.

Pearlman, L. A. y Saakvitne, K. W. (1995). Treating therapist with vicarious traumatization and secondary traumatic stress disorders. En C. R. Figley (Ed.), Compassion fatigue: Coping with secondary traumatic stress disorder in those who treat the traumatized (pp. 150-177). New York: Brunner/Mazel.

Richardson, J. I. (2001). Guidebook on vicarious trauma: Recommended solutions for anti-violence workers. Ottawa, ON: Health Canada.

Saakvitne, K. W. y Pearlman, L. A. (1996). Transforming the pain: A workbook on vicarious traumatization for helping professionals who work with traumatized clients. New York: W. W. Norton and Company Inc.

Sabo, M. (2006). Compassion fatigue and nursing work: Can we accurately capture the consequences of caring work?. International Journal of Nursing Practice 12, 136-142.

Salston, M. D. y Figley, C. R. (2003). Secondary traumatic stress: Effects of working with survivors of criminal victimization. Journal of Traumatic Stress, 16, 167-174.

Valent, P. (2002). Diagnosis and treatment of helper stresses, traumas, and illnesses. En C. R. Figley (Ed.), Treating compassion fatigue (pp. 17-37). New York: Brunner-Routledge. 